\title{
Genetic Polymorphisms of miR-146a and miR-27a, H. pylori Infection, and Risk of Gastric Lesions in a Chinese Population
}

\author{
Ming-yang Song ${ }^{1}$, Hui-juan Su ${ }^{1}$, Lian Zhang ${ }^{1}$, Jun-ling Ma ${ }^{1}$, Ji-you $\mathrm{Li}^{2}$, Kai-feng Pan ${ }^{1 *}$, Wei-cheng You ${ }^{1 *}$ \\ 1 Key Laboratory of Carcinogenesis and Translational Research (Ministry of Education), Department of Cancer Epidemiology, Peking University Cancer Hospital and \\ Institute, Beijing, People's Republic of China, 2 Department of Pathology, Peking University Cancer Hospital and Institute, Beijing, People's Republic of China
}

\begin{abstract}
Background: MicroRNAs (miRNAs) have been implicated in various human diseases. Single nucleotide polymorphisms (SNPs) in inflammation-related miRNA may play an important role in Helicobacter pylori (H. pylori)-induced gastric lesions. To evaluate the associations between miRNA SNPs, H. pylori and gastric lesions, a population-based study was conducted in Linqu County, China.

Methodology/Principal Findings: Based on serum miRNA array conducted in this population, two SNP loci (miR-146a rs2910164: $G>C$ and miR-27a rs895819: T>C) were determined by polymerase chain reaction-restriction fragment length polymorphism in 2,380 participants with diverse gastric lesions. Using participants with superficial gastritis and mild chronic atrophic gastritis as the reference group, we found that rs2910164 CC carriers had a significantly increased risk of intestinal metaplasia [adjusted odds ratio (OR), 1.42; 95\% confidence interval (Cl), 1.03-1.97] and dysplasia (OR, 1.54; 95\% Cl, 1.052.25) compared to GG carriers, whereas no significant association was observed for rs895819. Stratified analysis by $H$. pylori infection indicated that rs2910164 C allele was associated with an increased risk of intestinal metaplasia and dysplasia only among individuals infected with $\mathrm{H}$. pylori (CC vs. GG: OR, 1.53; $95 \% \mathrm{Cl}, 1.12-2.08, P$ for trend $=0.004$ ). Participants who simultaneously carried variant alleles and $H$. pylori infection were more likely to develop intestinal metaplasia and dysplasia, although the interaction between genetic variants and $H$. pylori infection was not significant $(P$ for interaction $=0.35$ for rs2910164 and 0.92 for rs895819).
\end{abstract}

Conclusions/Significance: These findings suggest that miR-146a rs2910164 polymorphism may contribute to the evolution of $H$. pylori-associated gastric lesions in this high-risk population.

Citation: Song M-y, Su H-j, Zhang L, Ma J-I, Li J-y, et al. (2013) Genetic Polymorphisms of miR-146a and miR-27a, H. pylori Infection, and Risk of Gastric Lesions in a Chinese Population. PLoS ONE 8(4): e61250. doi:10.1371/journal.pone.0061250

Editor: Hiromu Suzuki, Sapporo Medical University, Japan

Received January 7, 2013; Accepted March 7, 2013; Published April 17, 2013

Copyright: (C) 2013 Song et al. This is an open-access article distributed under the terms of the Creative Commons Attribution License, which permits unrestricted use, distribution, and reproduction in any medium, provided the original author and source are credited.

Funding: This work was supported by grants from the National Basic Research Program of China (973 Program: 2010CB529303), A3 Foresight Program from Natural Science Foundation of China (30921140311), National Natural Science Foundation of China (81171989), and the Key International S\&T Cooperation Project (2010DFB30310). The funders had no role in study design, data collection and analysis, decision to publish, or preparation of the manuscript.

Competing Interests: The authors have declared that no competing interests exist.

*E-mail: weichengyou@yahoo.com (WY); pankaifeng2002@yahoo.com (KP)

\section{Introduction}

Helicobacter pylori $($ H. pylor $)$ is a gram-negative bacterial pathogen and has been recognized as the major cause of gastric cancer $(\mathrm{GC})$ [1]. Chronic gastritis induced by H. pylori can persist for decades and may progress to intestinal metaplasia (IM), dysplasia (DYS) and intestinal-type GC [2]. Although about half of the world's population is infected with $H$. pylori, only a small proportion of infected people ultimately develop GC [3], suggesting that genetic polymorphisms in inflammation-related genes may play a role in this process $[4,5]$. Recent studies demonstrate that single nucleotide polymorphisms (SNPs) in microRNAs (miRNAs) could also affect cancer risk [6]

MiRNAs are an abundant class of small non-coding RNAs that involve in various biological processes and human diseases by negatively regulating the translational efficiency and stability of their target mRNAs [7-9]. Studies have shown that SNP or mutation in miRNA sequence may influence cancer susceptibility by altering miRNA expression, maturation or miRNA-mRNA interaction $[6,10,11]$. In recent years, we focused on the evaluation of circulating miRNAs in early detection of GC, and identified the differentially expressed miRNAs in GG through genome-wide serum miRNA expression profiling [12]. Based on this array data, we searched for potential functional SNP loci which could be involved in $H$. pylori-induced inflammatory process. Among them, we selected two SNPs for the current study, miR-146a rs2910164: $\mathrm{G}>\mathrm{C}$ and $m i R-27 a$ rs895819: $\mathrm{T}>\mathrm{C}$, which have been reported to influence GC risk [13,14].

MiR-146a is involved in the regulation of innate immunity and $H$. pylori-induced inflammatory response through modulating the expression of target genes, IL-1 receptor-associated kinase 1 (IRAK1) and TNF receptor-associated factor 6 (TRAF6) [15,16]. $m i R-27 a$ has been shown to function as oncogenes in gastric adenocarcinoma by targeting prohibitin and forkhead box protein Ol (FOXO1) [13,17], which could protect cells against oxidative stress $[18,19]$. Because $H$. pylori infection-induced inflammation is 
an important source of oxidative stress by producing reactive oxygen species (ROS), we also selected $m i R-27 a$ as a candidate. The polymorphisms of rs2910164 in miR-146a and rs895819 in $m i R-27 a$ have been reported to affect the corresponding miRNA production [20,21] and GC susceptibility [14,22]. However, there are limited data on their relationships with $H$. pylori-induced premalignant gastric lesions [23], particularly in a populationbased study.

Based on the above evidence, we performed genotyping analyses for $m i R-146 a$ and $m i R-27 a$ polymorphisms and evaluated their associations with various gastric lesions in 2,380 participants from a gastroscopy-based study in Linqu County, Shandong Province, a high-risk area of GC in China.

\section{Materials and Methods}

\section{Study Population}

From November 1989 through March 1990, a total of 3433 individuals participated in an endoscopic screening survey, representing $83 \%$ of eligible residents aged 35-64 years in 14 villages selected at random within four townships of Linqu County, as described previously [24]. The study was approved by the Institutional Review Boards of Peking University Cancer Hospital \& Institute, and all participants gave written informed consent. In the current study, a total of 2380 participants providing the blood samples and having gastric histopathologic diagnoses were included. There was no evident difference in basic characteristics between the included and excluded participants (data not shown).

\section{Histopathology}

Details of the gastroscopic procedures and histopathologic criteria have been described elsewhere [3,24]. Briefly, for each participant, biopsy samples were taken at seven standard sites in the stomach mucosa and given its corresponding histopathologic diagnosis by three senior pathologists independently. Each biopsy was classified according to the presence or absence of superficial gastritis (SG), chronic atrophic gastritis (CAG, mild or severe), IM (superficial or deep), DYS (mild, moderate or severe) or GC. Each biopsy was given a diagnosis based on the most severe histology, and each participant was assigned a global diagnosis based upon the most severe diagnosis among any of the biopsies.

\section{Blood Sample Collection and DNA Preparation}

A $5 \mathrm{ml}$ blood sample was collected from each participant, allowed to clot for $30-40 \mathrm{~min}$ at room temperature and then centrifuged at $965 \mathrm{~g}$ for $15 \mathrm{~min}$. The resulting serum was separated into vials. The clot and serum were stored immediately at $-20^{\circ} \mathrm{C}$ and then moved into a $-70^{\circ} \mathrm{C}$ freezer at Peking University Cancer Hospital \& Institute within 2 or 3 days after collection. High molecular weight genomic DNA was isolated by standard proteinase $\mathrm{K}$ digestion and phenol-chloroform extraction from the blood samples.

\section{H. pylori Antibody Assay}

As described previously, $H$. pylori antibody assays were used to determine $H$. pylori infection status in 1989 [25]. In brief, serum levels of anti- $H$. pylori $\mathrm{IgG}$ and $\mathrm{IgA}$ were measured separately in duplicate with enzyme-linked immunosorbent assay procedures. An individual was defined to be positive for $H$. pylori infection if the mean optical density for either IgG or IgA was $\geq 1.0$. Quality control samples were assayed at Vanderbilt University, Nashville, TN.

\section{Genotyping Analysis}

Genotyping was performed by polymerase chain reactionrestriction fragment length polymorphism (PCR-RFLP) analysis. Genomic DNA was amplified in a $20-\mu$ l reaction mixture, containing $100 \mathrm{ng}$ of template DNA, $10 \mathrm{pmol}$ of each primer (rs2910164: F: 5'-CATGGGTTGTGTCAGTGTCAGAGCT-3' and R: 5'-TGCCTTCTGTCTCGAGTCTTCCAA-3'; rs895819: F: 5'-GAACTTAGCGACTGTGAACACGACTTGG-3' and R: 5'-TTGCTTCCTGTCACAAATCACATTG-3'), $0.0625 \mathrm{mM}$ of each dNTP, $0.5 \mathrm{mM}$ of $\mathrm{MgCl}_{2}$ and $0.5 \mathrm{U}$ GoTaq ${ }^{\circledR}$ DNA polymerase in $5 \times$ reaction buffer (Promega, Madison, WI). PCR was accomplished by an initial denaturation of $95^{\circ} \mathrm{C}$ for $2 \mathrm{~min}$, followed by 35 cycles of $95^{\circ} \mathrm{C}$ for $30 \mathrm{~s}, 60^{\circ} \mathrm{C}$ for $45 \mathrm{~s}$ and $72^{\circ} \mathrm{C}$ for $45 \mathrm{~s}$, with a final elongation at $72^{\circ} \mathrm{C}$ for $5 \mathrm{~min}$.

For RFLP analysis, PCR products were digested with appropriate restriction endonucleases and then visualized using the Ultra Violet gel imaging system on a 3.5\% agarose gel that contained $0.5 \mu \mathrm{g} / \mathrm{mL}$ ethidium bromide. For rs2910264 genotyping, a $4.0 \mu \mathrm{l}$ aliquot of PCR products was digested with $1.0 \mu \mathrm{l}$ of FastDigest ${ }^{\circledR}$ Sac I in $10 \times$ FastDigest ${ }^{\circledR}$ Green Buffer (Fermentas) by incubation at $37^{\circ} \mathrm{C}$ for $30 \mathrm{~min}$ and inactivation at $65^{\circ} \mathrm{C}$ for $5 \mathrm{~min}$. The genotypes were assessed as follows: a single $147 \mathrm{bp}$ fragment for the GG genotype; two fragments of 122 and 25 bp for the CG genotype; and three fragments of 147, 122, and 25 bp for the GC genotype. For rs895819 genotyping, a $5.0 \mu \mathrm{l}$ aliquot of PCR products was digested with $0.5 \mu \mathrm{l}$ of FastDigest ${ }^{\circledR}$ Dra III in $10 \times$ FastDigest ${ }^{\circledR}$ Green Buffer (Fermentas, Burlington, Canada) by incubation at $37^{\circ} \mathrm{C}$ for $5 \mathrm{~min}$ and inactivation at $80^{\circ} \mathrm{C}$ for $5 \mathrm{~min}$. The genotypes were assessed as follows: a single $182 \mathrm{bp}$ fragment for the CG genotype; two fragments of 155 and 27 bp for the TT genotype; and three fragments of 182, 155, and 27 bp for the TC genotype. The genotypes identified by RFLP were further confirmed by DNA sequencing with ABI Prism 377 DNA Sequencer (Applied Biosystems, Foster City, CA).

\section{Quality Control Procedures}

Rigorous quality control procedures were applied throughout genotyping process. To avoid PCR contamination, reagents for PCR were carefully aliquoted and each aliquot was used no more than three times. A negative control (no DNA template) was added in each assay to monitor PCR contamination. A pilot study (50 samples) was conducted to optimize the conditions of PCR and restriction digestion. The electrophoretogram was read by one or two trained technicians blinded to the diagnosis of each participant and independent triplicate experiments were done for dubious samples. After genotyping, approximately $10 \%$ to $15 \%$ of samples in each genotype group were selected for repeated assays using PCR-DNA sequencing and the concordance rate was $>99 \%$.

\section{Statistical Analysis}

Because there were very few participants with normal gastric mucosa or SG in this population, we combined them with mild CAG as one group. Consequently, all participants were divided into four lesion groups: SG/mild CAG $(n=965)$, severe CAG $(\mathrm{n}=204), \mathrm{IM}(\mathrm{n}=765)$ and DYS $(\mathrm{n}=446)$. We used one-way analysis of variance to examine the overall difference in age, and Pearson's $\chi^{2}$ test to test the differences in distributions of categorical variables (i.e. gender, H. pylori infection, smoking, drinking and genotype) among groups. We employed a goodnessof-fit $\chi^{2}$ analysis to test the Hardy-Weinberg equilibrium.

To assess the associations of genetic variants with gastric lesions, we estimated odds ratios (ORs) and 95\% confidence intervals (CIs) using the unconditional multivariate logistic regression model with SG/mild CAG as the reference group, and adjusted for age, 
gender, H. pylori infection, smoking and drinking status. Test for trend was conducted in logistic regression under the codominant model by using a 3-level ordinal variable for each SNP ( 0 = homozygote wild, $1=$ heterozygote, $2=$ homozygote variant $)$.

To evaluate whether observed associations varied by $H$. pylori infection, we conducted stratified analyses using unconditional logistic regression. For gene - H. pylori infection joint effect analysis, a composite variable with values of 1 to 6 was generated by assembling levels of the two combined factors, and ORs and 95\% confidence intervals were estimated by adding this variable to the logistic model after transformation of dummy variables. Potential interactions between polymorphisms and $H$. pylori infection were evaluated on the multiplicative scale by adding a cross-product term between $H$. pylori infection and genotypes of each SNP into the multivariate logistic model. $P$ for interaction was calculated using the likelihood ratio test to compare the two models with and without interaction terms (degree of freedom $=2$ ).

All analyses were performed using the Statistical Analysis System software (version 9.1; SAS Institute, Cary, NC). $P$ value of $<0.05$ was considered significant and all statistical tests were twosided.

\section{Results}

\section{Participant Information}

A total of 2,380 participants (1,180 males and 1,200 females) were enrolled in this study, with the mean age of $45.3 \pm 8.2$ (Table 1). Information on $H$. pylori infection, smoking, and drinking status was available in 2,078 (87.3\%), 2,343 (98.4\%) and 2,339 (98.3\%) participants, respectively. Significant differences in the overall distributions of age, gender, $H$. pylori infection, smoking and drinking status were identified among four lesion groups. As expected, more elder, male, H. pylori-positive, smoking, and drinking persons were found with severe CAG, IM and DYS than with SG/mild CAG.

\section{Genotype Distributions}

The genotype distributions of the two polymorphisms in various gastric lesion groups are shown in Table 2. The genotype frequencies of these two SNPs in the study population fit the Hardy-Weinberg equilibrium $(P=0.08$ for rs2910164; $P=0.48$ for rs895819). Overall, no significant difference was observed in the genotype distributions of the two SNPs among different groups $(P=0.08$ for rs2910164; $P=0.91$ for rs895819).

\section{Associations of miRNA Polymorphisms with Risk of Gastric Lesions}

We firstly evaluated the association between the two SNPs and risk of advanced gastric lesions. As shown in Table 2, participants carrying rs2910164 CG genotype had a significantly increased risk of developing IM (OR, 1.42; 95\% CI, 1.03-1.97) and DYS (OR, 1.54; 95\% CI, 1.05-2.25) compared to GG genotype, after adjusting for age, gender, $H$. pylori infection, smoking and drinking status. The number of rs2910164 $\mathrm{C}$ allele showed a significant dose-response relationship with the risk of $\operatorname{IM}(P$ for trend $=0.02)$ and DYS $(P$ for trend $=0.01)$. No significant association was found for rs895819 and risk of advanced gastric lesions.

We further examined whether $H$. pylori infection modified the associations of SNPs with advanced gastric lesions by stratified analysis. We collapsed the four-category outcome into a binary variable to increase statistical power, and evaluated the association between genetic variants and the risk of IM/DYS in reference to SG/CAG. As shown in Table 3, among participants with positive H. pylori infection, those who carried rs2910164 CG genotype had a significantly increased risk of IM/DYS compared to GG carriers $(\mathrm{OR}, 1.53 ; 95 \%$ CI, $1.12-2.08, P$ for trend $=0.004)$, whereas such association was not found among participants without $H$. pylori infection (OR, 0.93; 95\% CI, 0.52-1.66, $P$ for trend $=0.90)$. For rs895819, no significant association was observed among individuals either with or without $H$. pylori infection.

Table 1. Selected characteristics of participants with various gastric lesions.

\begin{tabular}{|c|c|c|c|c|c|c|}
\hline Variable & $\begin{array}{l}\text { SG/mild CAG* } \\
n=965\end{array}$ & $\begin{array}{l}\text { Severe CAG* } \\
n=204\end{array}$ & $\begin{array}{l}I M^{*} \\
n=765\end{array}$ & $\begin{array}{l}\text { DYS* } \\
n=446\end{array}$ & $\begin{array}{l}\text { Total } \\
n=2,380\end{array}$ & $P^{\dagger}$ \\
\hline Mean age $\pm S D^{*}$, years & $43.5 \pm 7.4$ & $43.7 \pm 7.7$ & $46.4 \pm 8.5$ & $47.8 \pm 8.7$ & $45.3 \pm 8.2$ & $<0.001$ \\
\hline Gender (\%) & & & & & & $<0.001$ \\
\hline Male & $450(46.6)$ & $97(47.5)$ & $368(48.1)$ & $265(59.4)$ & $1,180(49.6)$ & \\
\hline Female & $515(53.4)$ & $107(52.5)$ & 397 (51.9) & 181 (40.6) & $1,200(50.4)$ & \\
\hline H. pylori infection (\%) & & & & & & $<0.001$ \\
\hline Positive & $527(54.6)$ & 159 (77.9) & 565 (73.9) & $340(76.2)$ & 1,591 (66.8) & \\
\hline Negative & $284(29.4)$ & $20(9.8)$ & $113(14.8)$ & 70 (15.7) & $487(20.5)$ & \\
\hline Missing & $154(16.0)$ & $25(12.3)$ & $87(11.3)$ & $36(8.1)$ & $302(12.7)$ & \\
\hline Smoking (\%) & & & & & & $<0.001$ \\
\hline Yes & $375(38.9)$ & $81(39.7)$ & $327(42.7)$ & $240(53.8)$ & $1,023(43.0)$ & \\
\hline No & $574(59.5)$ & $123(60.3)$ & $426(55.7)$ & $197(44.2)$ & $1,320(55.5)$ & \\
\hline Missing & $16(1.6)$ & $0(0.0)$ & $12(1.6)$ & $9(2.0)$ & $37(1.5)$ & \\
\hline Drinking (\%) & & & & & & 0.001 \\
\hline Yes & $420(43.5)$ & 87 (42.6) & $324(42.4)$ & $236(52.9)$ & $1,067(44.8)$ & \\
\hline No & $528(54.7)$ & $117(57.4)$ & 426 (55.6) & $201(45.1)$ & $1,272(53.4)$ & \\
\hline Missing & $17(1.8)$ & $0(0.0)$ & $15(2.0)$ & $9(2.0)$ & 41 (1.8) & \\
\hline
\end{tabular}

*SG = superficial gastritis; CAG = chronic atrophic gastritis; IM = intestinal metaplasia; DYS=dysplasia; SD = standard deviation.

${ }^{\dagger} P$ value for each covariate was estimated among participants without missing value on that covariate.

doi:10.1371/journal.pone.0061250.t001 
Table 2. Genotype distribution and associations of genetic variants with risk of gastric lesions.

\begin{tabular}{|c|c|c|c|c|c|c|c|}
\hline \multirow[t]{2}{*}{ Variant } & \multirow{2}{*}{$\begin{array}{l}\text { SG/mild CAG* } \\
\text { (\%) }\end{array}$} & \multicolumn{2}{|c|}{ Severe CAG* } & \multicolumn{2}{|l|}{$\mathrm{IM}^{*}$} & \multicolumn{2}{|l|}{ DYS* } \\
\hline & & n (\%) & OR $(95 \% \mathrm{Cl})^{* \dagger}$ & n (\%) & OR $(95 \% \mathrm{Cl})^{* \dagger}$ & n (\%) & OR $(95 \% \mathrm{CI})^{* \dagger}$ \\
\hline \multicolumn{8}{|l|}{ rs $2910164^{\ddagger}$} \\
\hline GG, n (\%) & $177(18.4)$ & $30(14.8)$ & 1.00 (referent) & $124(16.3)$ & 1.00 (referent) & 75 (16.8) & 1.00 (referent) \\
\hline GC, n (\%) & $511(53.1)$ & $104(51.2)$ & $1.24(0.77-1.99)$ & $374(49.1)$ & $1.14(0.84-1.54)$ & $212(47.5)$ & $1.09(0.76-1.55)$ \\
\hline CC, n (\%) & $275(28.5)$ & $69(34.0)$ & $1.40(0.84-2.34)$ & $264(34.6)$ & $1.42(1.03-1.97)$ & $159(35.7)$ & $1.54(1.05-2.25)$ \\
\hline$P$ for trend $d^{\S}$ & & & 0.21 & & 0.02 & & 0.01 \\
\hline \multicolumn{8}{|l|}{ rs895819" } \\
\hline $\mathrm{TT}, \mathrm{n}(\%)$ & $504(52.4)$ & $98(48.3)$ & 1.00 (referent) & $402(53.0)$ & 1.00 (referent) & $224(50.6)$ & 1.00 (referent) \\
\hline TC, n (\%) & $389(40.5)$ & $88(43.3)$ & $1.33(0.94-1.88)$ & $300(39.6)$ & $1.06(0.84-1.33)$ & $185(41.8)$ & $1.19(0.91-1.56)$ \\
\hline CC, n (\%) & $68(7.1)$ & $17(8.4)$ & $1.11(0.59-2.11)$ & $56(7.4)$ & $0.94(0.62-1.44)$ & $34(7.7)$ & $1.05(0.64-1.73)$ \\
\hline$P$ for trend $d^{\S}$ & & & 0.11 & & 0.65 & & 0.20 \\
\hline
\end{tabular}

* $\mathrm{SG}=$ superficial gastritis; $\mathrm{CAG}=$ chronic atrophic gastritis; $\mathrm{IM}=$ intestinal metaplasia; $\mathrm{DYS}=$ dysplasia; OR=odds ratio; $\mathrm{Cl}=\mathrm{confidence}$ interval.

${ }^{\dagger}$ Odds ratios and $95 \%$ confidence intervals were estimated in reference to SG/mild CAG group, and adjusted for age, gender, $H$. pylori infection, smoking and drinking status. The analysis was restricted to participants with complete data on all covariates.

‡Six participants with missing values on rs2910164 genotype were excluded from analysis.

${ }^{\S} P$ for trend were calculated by including the 3 -level ordinal variable under codominant model for each polymorphism $(0=$ homozygote wild, $1=$ heterozygote,

$2=$ homozygote variant) as a continuous variable to the multivariate models.

"Fifteen participants with missing values on rs895819 genotype were excluded from analysis.

doi:10.1371/journal.pone.0061250.t002

\section{Gene-H. pylori Infection Joint Effect and Interaction}

We also explored the joint effects and possible gene-environment interaction between these two SNPs and H. pylori infection. As shown in Table 4, compared to participants who were $H$. pylorinegative and carried homogeneous wild genotype of each SNP, participants with $H$. pylori infection alone or carrying both hazard allele and $H$. pylori infection had increased risk of advanced gastric lesions.
For rs2910164, individuals who simultaneously carried CC genotype and had $H$. pylori infection were at a 2.25 -fold increased risk of developing IM/DYS compared to those carrying GG genotype and without $H$. pylori infection (95\% CI, 1.43-3.54, $P$ for trend $<0.001)$. Similarly, for rs895819, the coexistence of CC genotype and $H$. pylori infection was associated with a higher risk of IM/DYS (OR, 2.77; 95\% CI, 1.68-4.56, $P$ for trend $<0.001$ ). However, the formal test for gene- $H$. pylori infection interaction

Table 3. Associations of genetic variants with risk of advanced gastric lesions by $H$. pylori infection*.

\begin{tabular}{|c|c|c|c|c|c|c|}
\hline \multirow{2}{*}{ Variant } & \multirow{2}{*}{ H. pylori infection } & \multirow{2}{*}{ Genotype } & \multirow{2}{*}{$\frac{\text { SG/CAG }{ }^{\dagger}}{n(\%)}$} & \multicolumn{3}{|l|}{ IM/DYS ${ }^{\dagger}$} \\
\hline & & & & n (\%) & OR $(95 \% \mathrm{Cl})^{\dagger \ddagger}$ & $P$ for trend $\mathrm{d}^{\mathrm{s}}$ \\
\hline \multirow[t]{6}{*}{ rs2910164" } & Negative & GG & $46(15.2)$ & $32(17.6)$ & 1.00 (referent) & \\
\hline & & GC & 165 (54.5) & $91(50.0)$ & $0.86(0.50-1.47)$ & \\
\hline & & CC & $92(30.4)$ & 59 (32.4) & $0.93(0.52-1.66)$ & 0.90 \\
\hline & Positive & GG & $124(18.1)$ & $145(16.1)$ & 1.00 (referent) & \\
\hline & & GC & 359 (52.4) & $444(49.2)$ & $1.15(0.86-1.53)$ & \\
\hline & & CC & $202(29.5)$ & $314(34.8)$ & $1.53(1.12-2.08)$ & 0.004 \\
\hline \multirow[t]{6}{*}{ rs895819 } & Negative & $\pi$ & $155(51.2)$ & $92(50.6)$ & 1.00 (referent) & \\
\hline & & TC & $129(42.6)$ & $77(42.3)$ & $1.03(0.69-1.54)$ & \\
\hline & & CC & $19(6.3)$ & $13(7.1)$ & $1.12(0.50-2.51)$ & 0.79 \\
\hline & Positive & $\pi$ & $358(52.5)$ & $472(52.7)$ & 1.00 (referent) & \\
\hline & & TC & $268(39.3)$ & $357(39.8)$ & $1.04(0.84-1.29)$ & \\
\hline & & CC & $56(8.2)$ & $67(7.5)$ & $0.92(0.62-1.36)$ & 0.94 \\
\hline
\end{tabular}

*This analysis was restricted to 2078 participants who had diagnosis for H. pylori infection.

${ }^{\dagger} \mathrm{SG}=$ superficial gastritis; $\mathrm{CAG}=$ chronic atrophic gastritis; $\mathrm{IM}=$ intestinal metaplasia; $\mathrm{DYS}=$ dysplasia; $\mathrm{OR}=$ odds ratio; $\mathrm{Cl}=$ confidence interval.

${ }^{\ddagger}$ Odds ratios and $95 \%$ confidence intervals were estimated in reference to SG/CAG group, and adjusted for age, gender, smoking and drinking status. Participants with missing data on any of covariates were excluded from the analysis.

${ }^{\S} P$ for trend was calculated by including the 3 -level ordinal variable under codominant model for each genetic variant $(0=$ homozygote wild, $1=$ heterozygote,

$2=$ homozygote variant) as a continuous variable to the multivariate models.

"Five participants with missing values on rs2910164 genotype were excluded from analysis.

"Fifteen participants with missing values on rs895819 genotype were excluded from analysis.

doi:10.1371/journal.pone.0061250.t003 
Table 4. Joint effect and interaction between polymorphisms and $H$. pylori infection on risk of intestinal metaplasia and dysplasia*.

\begin{tabular}{|c|c|c|c|c|}
\hline Variant & Genotype & $\begin{array}{l}\text { H. pylori } \\
\text { infection }\end{array}$ & OR $(95 \% \mathrm{Cl})^{\dagger}$ & $\begin{array}{l}P \text { for } \\
\text { trend }^{\ddagger}\end{array}$ \\
\hline \multirow[t]{7}{*}{ rs2910164 } & GG & Negative & 1.00 (referent) & \\
\hline & $\mathrm{GC}$ & Negative & $1.01(0.68-1.50)$ & \\
\hline & $\mathrm{CC}$ & Negative & $1.08(0.48-2.41)$ & \\
\hline & GG & Positive & $2.44(1.80-3.31)$ & \\
\hline & GC & Positive & $2.55(1.86-3.49)$ & \\
\hline & $\mathrm{CC}$ & Positive & $2.25(1.43-3.54)$ & $<0.001$ \\
\hline & $\begin{array}{l}P \text { for } \\
\text { interaction }^{\S}\end{array}$ & & 0.35 & \\
\hline \multirow[t]{7}{*}{ rs895819 } & $\pi$ & Negative & 1.00 (referent) & \\
\hline & $\mathrm{TC}$ & Negative & $0.87(0.51-1.48)$ & \\
\hline & $\mathrm{CC}$ & Negative & $0.93(0.52-1.66)$ & \\
\hline & $\pi$ & Positive & $1.83(1.09-3.10)$ & \\
\hline & $\mathrm{TC}$ & Positive & $2.09(1.28-3.39)$ & \\
\hline & $\mathrm{CC}$ & Positive & $2.77(1.68-4.56)$ & $<0.001$ \\
\hline & $\begin{array}{l}P \text { for } \\
\text { interaction }^{\S}\end{array}$ & & 0.92 & \\
\hline
\end{tabular}

*This analysis was restricted to 2078 participants who had diagnosis for $\mathrm{H}$. pylori infection.

†Odds ratios (ORs) and 95\% confidence intervals (Cl) for the risk of intestinal metaplasia and dysplasia were estimated in reference to superficial gastritis/ chronic atrophic gastritis, and adjusted for age, gender, smoking and drinking status. Participants with missing data on any of variables were excluded from the analysis.

${ }^{\ddagger} P$ for trend was calculated by assigning an ordinal value (1-6) to each combination of genotype and $\mathrm{H}$. pylori infection $(1=$ homozygote wild without $H$. pylori infection, $2=$ heterozygote without $H$. pylori infection, $3=$ homozygote variant without $H$. pylori infection, $4=$ homozygote wild with $H$. pylori infection, $5=$ heterozygote with $H$. pylori infection, $6=$ homozygote variant with $H$. pylori infection) and adding it as a continuous variable to the multivariate model.

${ }^{\S}$ Likelihood ratio test was used to calculate the $P$ value for interaction by comparing the two models with and without the product term of genotypes and $H$. pylori infection.

doi:10.1371/journal.pone.0061250.t004

did not attain statistical significance for either rs2910164 or rs895819 $(P$ for interaction $=0.35$ and 0.92 , respectively).

\section{Discussion}

In the present study, based on our previous miRNA array data, we selected two potential functional SNPs (rs2910164 in miR-146a and rs895819 in $m i R-27 a$ ) and investigated their relationship with H. pylori-associated gastric lesions in a Chinese population. We found that rs2910164 may affect the susceptibility of gastric lesions. To our best knowledge, this is the first study to evaluate the associations between miRNA polymorphisms and precancerous gastric lesions in a large population. Our findings suggest that genetic variants of miRNA may play a role in $H$. pylori-related gastric pathogenesis.

The rs2910164 $\mathrm{G}>\mathrm{C}$ polymorphism is located in the stem region opposite to the mature miR-146a sequence and results in a change from $\mathrm{G}: \mathrm{U}$ pair to $\mathrm{C}: \mathrm{U}$ mismatch in the stem structure of miR-146a precursor [11]. Several studies have reported the associations between rs2910164 and multiple malignancies, including prostate, hepatocellular, papillary thyroid and esophageal squamous cell carcinoma $[11,21,26,27]$. As for GC, the results have been mixed. A Chinese study without adjusting for H. pylori infection showed that persons carrying rs2910164 GG/GC genotype were at significantly increased risk of GC compared to CC genotype [14], while a Japanese study demonstrated that individuals carrying $\mathrm{C}$ allele were more likely to develop gastric carcinoma than GG carriers after adjusting for $H$. pylori infection [22]. However, a study in the Korean population did not observe a significant association between rs2910164 and risk of GC [28]. A recent meta-analysis found that rs2910164 GG/GC genotype was significantly associated with increased overall cancer susceptibility in Asians [29]. Our results are consistent with the Japanese report, suggesting that rs2910164 CG carriers had a higher risk of IM and DYS after controlling for potential confounders including $H$. pylori infection. We also found that the association between rs2910164 CC genotype and risk of IM and DYS appeared to be more pronounced in $H$. pylori-positive individuals.

MiR-146a has been implicated in control of toll-like receptors (TLRs) and cytokine signaling pathway, reducing NF- $\kappa \mathrm{B}$ activity through negatively regulating IRAK 1 and TRAF6 expressions [16,30]. It's well known that TLR2, 4, 5 and 9 are involved in $H$. pylori recognition [31] and $\mathrm{NF}-\kappa \mathrm{B}$ is a key molecule in inflammation-cancer link [32]. Several studies provided the experimental evidences that rs2910164 $\mathrm{C}$ allele in miR-146 precursor could reduce mature miR-146a production [11,21], which might modify the inflammatory process. Although the exact mechanism of miR-146a polymorphism on the $H$. pylori-associated pathogenesis is unclear, it is possible that rs2910164 $\mathrm{G}>\mathrm{C}$ polymorphism might interfere the normal immune response to $H$. pylori infection and thus contribute to the elevated risk of advanced gastric lesions, such as IM and DYS. The functional relevance of this polymorphism as well as the exact mechanism in the $H$. pylori-associated carcinogenesis needs to be confirmed.

The rs895819 $\mathrm{T}>\mathrm{C}$ polymorphism is located in the $m i R-27 a$ precursor, at position 40 relative to the first nucleotide. $M i R-27 a$ is located at chromosome 19 and has been shown to function as oncogene by targeting prohibitin [13], FOXO1 [17] and Sprouty2 [33] in various cancers including GC. Accumulating evidence from basic research suggests that prohibitin and FOXOl may play important roles in inflammatory process by modulating early inflammatory responses [34] and protecting inflammation-associated oxidative injury $[18,35,36]$. However, epidemiologic evidence on the association between rs895819 polymorphism and GC and precancerous gastric lesions is sparse [20,23,37].

Recently, a Chinese study found that rs895819 TG/CG genotype was associated with an increased risk of $\mathrm{GC}$ by enhancing miR-27a production and reducing the mRNA level of its target gene ZBTB10 [20]. However, this association was not replicated by another study [37]. A Japanese study examined the relationship between rs895819 and chronic gastritis in 179 participants without gastric malignancy, and observed that $\mathrm{CC}$ carriers had significantly higher atrophy and metaplasia scores than TG/TT carriers in men [23]. In the current study, we did not observe a significant association between rs895819 and risk of gastric lesions. Given the sparse literature [38], however, further studies are needed to investigate the relationship of rs895819 with gastric carcinogenesis.

We were also interested to evaluate the possible joint effect and interaction between $\mathrm{rs} 2910164 \mathrm{G}>\mathrm{C}$ or $\operatorname{rs895819} \mathrm{T}>\mathrm{C}$ polymorphism and $H$. pylori infection. A significantly increased risk of IM and DYS was observed in participants with $H$. pylori infection alone, consistent with our previous finding in Linqu that $H$. pylori infection played an important role in the incidence and progression of gastric lesions [25]. Further analysis revealed a significant joint effect between rs2910164, rs895819 and H. pylori infection, suggesting a gene-environmental interplay may modify 
H. pylori-related carcinogenesis and outcome of $H$. pylori infection. However, given the limited sample size, the formal tests failed to detect any significant interaction. Since successful identification of gene- $H$. pylori interaction could help discern the intricate mechanism of gastric carcinogenesis and explain the high variation in the GC incidence observed both around the world and within the high $H$. pylori prevalence population, more and larger population studies are deserved in this area [39].

Our study has both strengths and limitations. The strengths of this study include the relatively large homogeneous population, well-defined histopathological diagnosis, and detailed information on potential confounders, especially $H$. pylori infection. In addition, we selected the potential functional SNP loci from the differentially expressed miRNAs based on the serum profiling of GC. This approach increased the possibility that our identified SNP loci were biologically relevant to gastric carcinogenesis.

One limitation of this study was the combination of SG/mild CAG as controls in overall analysis because very few participants were diagnosed with normal gastric mucosa in this population [24]. However, this "sub-normal" control could only lead to the dilution of disparity between comparison groups and in turn attenuate the magnitude of association. In addition, we converted

\section{References}

1. Parsonnet J, Friedman GD, Vandersteen DP, Chang Y, Vogelman JH, et al, (1991) Helicobacter pylori infection and the risk of gastric carcinoma. N Engl J Med 325: 1127-1131.

2. Correa P (1988) A human model of gastric carcinogenesis. Cancer Research 48: 3554-3560.

3. You WC, Li JY, Blot WJ, Chang YS, Jin ML, et al. (1999) Evolution of precancerous lesions in a rural Chinese population at high risk of gastric cancer. Int J Cancer 83: 615-619.

4. Zeng HM, Pan KF, Zhang Y, Zhang L, Ma JL, et al. (2011) Genetic variants of toll-like receptor 2 and 5, helicobacter pylori infection, and risk of gastric cancer and its precursors in a chinese population. Cancer epidemiology, biomarkers \& prevention : a publication of the American Association for Cancer Research, cosponsored by the American Society of Preventive Oncology 20: 2594-2602.

5. Lu W, Pan K, Zhang L, Lin D, Miao X, et al. (2005) Genetic polymorphisms of interleukin (IL)-1B, IL-1RN, IL-8, IL-10 and tumor necrosis factor $\{$ alpha $\}$ and risk of gastric cancer in a Chinese population. Carcinogenesis 26: 631-636.

6. Ryan BM, Robles AI, Harris CG (2010) Genetic variation in microRNA networks: the implications for cancer research. Nat Rev Cancer 10: 389-402.

7. Bartel DP (2004) MicroRNAs: genomics, biogenesis, mechanism, and function. Cell 116: 281-297.

8. Esquela-Kerscher A, Slack FJ (2006) Oncomirs - microRNAs with a role in cancer. Nat Rev Cancer 6: 259-269.

9. Alvarez-Garcia I, Miska EA (2005) MicroRNA functions in animal development and human disease. Development 132: 4653-4662.

10. Yang Q Jie Z, Ye S, Li Z, Han Z, et al. (2012) Genetic variations in miR-27a gene decrease mature miR-27a level and reduce gastric cancer susceptibility. Oncogene.

11. Jazdzewski K, Murray EL, Franssila K, Jarzab B, Schoenberg DR, et al. (2008) Common SNP in pre-miR-146a decreases mature miR expression and predisposes to papillary thyroid carcinoma. Proc Natl Acad Sci U S A 105: $7269-7274$.

12. Song MY, Pan KF, Su HJ, Zhang L, Ma JL, et al. (2012) Identification of Serum MicroRNAs as Novel Non-Invasive Biomarkers for Early Detection of Gastric Cancer. PLoS One 7: e33608.

13. Liu T, Tang H, Lang Y, Liu M, Li X (2009) MicroRNA-27a functions as an oncogene in gastric adenocarcinoma by targeting prohibitin. Cancer Lett 273 : 233-242.

14. Zeng Y, Sun QM, Liu NN, Dong GH, Chen J, et al. (2010) Correlation between pre-miR-146a $\mathrm{C} / \mathrm{G}$ polymorphism and gastric cancer risk in Chinese population. World J Gastroenterol 16: 3578-3583.

15. Liu Z, Xiao B, Tang B, Li B, Li N, et al. (2010) Up-regulated microRNA-146a negatively modulate Helicobacter pylori-induced inflammatory response in human gastric epithelial cells. Microbes Infect 12: 854-863.

16. Taganov KD, Boldin MP, Chang KJ, Baltimore D (2006) NF-kappaBdependent induction of microRNA miR-146, an inhibitor targeted to signaling proteins of innate immune responses. Proc Natl Acad Sci U S A 103: 1248112486.

17. Guttilla IK, White BA (2009) Coordinate regulation of FOXO1 by miR-27a, miR-96, and miR-182 in breast cancer cells. J Biol Chem 284: 23204-23216.

18. Theiss AL, Vijay-Kumar M, Obertone TS, Jones DP, Hansen JM, et al. (2009) Prohibitin is a novel regulator of antioxidant response that attenuates colonic inflammation in mice. Gastroenterology 137: 199-208, 208 e191-196. the outcome from four categories into a binary variable (IM/DYS vs. SG/CAG) to preserve statistical power in stratification and interaction analysis, which may lead to outcome misclassification and loss of information. Another potential drawback is that we did not analyze the SNPs in the target genes of these miRNAs. Since miRNAs indirectly play biological roles through regulating target mRNA expression, SNPs in both miRNA and its target genes may interactively affect the progression of precancerous gastric lesions.

In summary, our population-based study provided evidence that rs2910164 polymorphism in $m i R-146$ was associated with advanced gastric lesions. Rs2910164 polymorphism might promote the occurrence of intestinal metaplasia and dysplasia jointly with $H$. pylori infection. Further functional validation of these observational findings needs to be conducted.

\section{Author Contributions}

Conceived and designed the experiments: WCY KFP. Performed the experiments: MYS HJS LZ JLM JYL. Analyzed the data: WCY KFP MYS HJS. Contributed reagents/materials/analysis tools: WCY KFP. Wrote the paper: WCY KFP MYS HJS.

19. Furukawa-Hibi Y, Yoshida-Araki K, Ohta T, Ikeda K, Motoyama N (2002) FOXO forkhead transcription factors induce $\mathrm{G}(2)-\mathrm{M}$ checkpoint in response to oxidative stress. J Biol Chem 277: 26729-26732.

20. Sun Q, Gu H, Zeng Y, Xia Y, Wang Y, et al. (2010) Hsa-mir-27a genetic variant contributes to gastric cancer susceptibility through affecting miR-27a and target gene expression. Cancer Sci 101: 2241-2247.

21. Xu T, Zhu Y, Wei QK, Yuan Y, Zhou F, et al. (2008) A functional polymorphism in the miR-146a gene is associated with the risk for hepatocellular carcinoma. Carcinogenesis 29: 2126-2131.

22. Okubo M, Tahara T, Shibata T, Yamashita H, Nakamura M, et al. (2010) Association between common genetic variants in pre-microRNAs and gastric cancer risk in Japanese population. Helicobacter 15: 524-531.

23. Arisawa T, Tahara T, Shibata T, Nagasaka M, Nakamura M, et al. (2007) A polymorphism of microRNA 27 a genome region is associated with the development of gastric mucosal atrophy in Japanese male subjects. Dig Dis Sci 52: 1691-1697.

24. You WC, Blot WJ, LiJY, Chang YS, Jin ML, et al. (1993) Precancerous gastric lesions in a population at high risk of stomach cancer. Cancer Res 53: 13171321.

25. Zhang L, Blot WJ, You WC, Chang YS, Kneller RW, et al. (1996) Helicobacter pylori antibodies in relation to precancerous gastric lesions in a high-risk Chinese population. Cancer Epidemiol Biomarkers Prev 5: 627-630.

26. Xu B, Feng NH, Li PC, Tao J, Wu D, et al. (2010) A functional polymorphism in Pre-miR-146a gene is associated with prostate cancer risk and mature miR146a expression in vivo. Prostate 70: 467-472.

27. Guo H, Wang K, Xiong G, Hu H, Wang D, et al. (2010) A functional varient in microRNA-146a is associated with risk of esophageal squamous cell carcinoma in Chinese Han. Fam Cancer 9: 599-603.

28. Ahn DH, Rah H, Choi YK, Jeon YJ, Min KT, et al. (2012) Association of the miR-146aC $>$ G, miR-149T $>$ C, miR-196a2T $>$ C, and miR-499A $>$ G polymorphisms with gastric cancer risk and survival in the Korean population. Molecular carcinogenesis

29. Wang J, Bi J, Liu X, Li K, Di J, et al. (2012) Has-miR-146a polymorphism (rs2910164) and cancer risk: a meta-analysis of 19 case-control studies. Molecular biology reports 39: 4571-4579.

30. Bhaumik D, Scott GK, Schokrpur S, Patil CK, Campisi J, et al. (2008) Expression of microRNA-146 suppresses NF-kappaB activity with reduction of metastatic potential in breast cancer cells. Oncogene 27: 5643-5647.

31. Akira S, Takeda K (2004) Toll-like receptor signalling. Nat Rev Immunol 4: 499-511.

32. Karin M (2006) Nuclear factor-kappaB in cancer development and progression. Nature 441: 431-436.

33. Ma Y, Yu S, Zhao W, Lu Z, Chen J (2010) miR-27a regulates the growth, colony formation and migration of pancreatic cancer cells by targeting Sprouty2. Cancer Lett 298: 150-158.

34. Sharma A, Qadri A (2004) Vi polysaccharide of Salmonella typhi targets the prohibitin family of molecules in intestinal epithelial cells and suppresses early inflammatory responses. Proc Natl Acad Sci U S A 101: 17492-17497.

35. Theiss AL, Idell RD, Srinivasan S, Klapproth JM, Jones DP, et al. (2007) Prohibitin protects against oxidative stress in intestinal epithelial cells. Faseb J 21: 197-206. 
36. Theiss AL, Jenkins AK, Okoro NI, Klapproth JM, Merlin D, et al. (2009) Prohibitin inhibits tumor necrosis factor alpha-induced nuclear factor-kappa B nuclear translocation via the novel mechanism of decreasing importin alpha3 expression. Mol Biol Cell 20: 4412-4423.

37. Zhou Y, Du WD, Chen G, Ruan J, Xu S, et al. (2012) Association analysis of genetic variants in microRNA networks and gastric cancer risk in a Chinese Han population. Journal of cancer research and clinical oncology 138: 939-945.
38. Zhong S, Chen Z, Xu J, Li W, Zhao J (2012) Pre-mir-27a rs895819 polymorphism and cancer risk: a meta-analysis. Molecular biology reports.

39. Gonzalez CA, Sala N, Capella G (2002) Genetic susceptibility and gastric cancer risk. Int J Cancer 100: 249-260. 